\title{
A Class of Partial Integro-Differential Equations with Correlation-Convolution Integral I
}

\author{
L. von Wolfersdorf
}

Dedicated to Prof. W. Krabs on the occasion of his 70th birthday

\begin{abstract}
By means of the iteration method with weighted norms existence and uniqueness theorems are proved for three classes of nonlinear integral equations and first order integro-differential equations in two variables. The quadratic nonlinearity is given by the correlation-convolution integral. Existence and uniqueness of the solutions are shown in Lebesgue spaces with mixed norms in rectangle and strip.

Keywords: Nonlinear integral equation, nonlinear partial integro-differential equations, correlation-convolution integral, iteration method with weighted norms
\end{abstract}

AMS subject classification: Primary 45G10, 45K05, secondary 44A30

\section{Introduction}

The paper deals with a class of integral equations and two classes of first order integro-differential equations in two variables with a quadratic integral term composed by an auto-convolution with respect to one variable and an auto-correlation with respect to the other variable. These equations generalize integral and first order integro-differential equations in one variable with an auto-convolution term (as appearing in equations for reflection functions in linear wave theorie $[1,8]$ ) on the one side and with an auto-correlation term (as appearing in the equations of statistical mechanics [4 - 7, 9]) on the other side. The integro-differential equations may be considered as model equations for general partial integro-differential equations with correlation-convolution integrals.

L. von Wolfersdorf: TU Bergakademie Freiberg, Fak. für Math. \& Inf., D-09596 Freiberg; wolfersd@math.tu-freiberg.de

ISSN 0232-2064 / \$2.50 C Heldermann Verlag Berlin 
In this first part of the paper, for equations in rectangle and strip the iteration method in Banach spaces with weighted norms is used for proving existence and uniqueness of solutions in Lebesgue spaces with mixed norms. Also, by means of exponential weights, uniqueness of the solutions to the equations is discussed in a direct manner. In the second part of the paper, for equations in the quarter plane, existence and constructive formulas for solutions will be investigated by means of methods of complex function theory.

\section{Statement of equations}

We deal with the integral and integro-differential equations in two variables

$$
\begin{array}{r}
p(t, \tau)+I_{0}[p](t, \tau)=h(t, \tau) \\
\frac{\partial p}{\partial \tau}+\mu p(t, \tau)+I_{0}[p](t, \tau)=h(t, \tau) \\
\frac{\partial p}{\partial t}+\lambda \frac{\partial p}{\partial \tau}+\mu p(t, \tau)+I_{0}[p](t, \tau)=h(t, \tau)
\end{array}
$$

for $(t, \tau) \in R:=\left(0, T_{0}\right) \times(0, T)$ with $0<T_{0}, T<\infty$, where $\lambda, \mu \in \mathbb{R}$ and $I_{0}$ is the quadratic correlation-convolution operator of $p$

$$
\begin{aligned}
I_{0}[p](t, \tau) & =\int_{0}^{\tau} \int_{0}^{T_{0}-t} p(s, \sigma) p(s+t, \tau-\sigma) d s d \sigma \\
& =\int_{0}^{\tau} \int_{0}^{T_{0}-t} p(s, \tau-\sigma) p(s+t, \sigma) d s d \sigma .
\end{aligned}
$$

These equations are also considered for $(t, \tau) \in S:=(0, \infty) \times(0, T)$ (i.e. for $\left.T_{0}=\infty\right)$, were the integral $I_{0}$ is replaced by

$$
I[p](t, \tau)=\int_{0}^{\tau} \int_{0}^{\infty} p(s, \sigma) p(s+t, \tau-\sigma) d s d \sigma .
$$

To equation (2) and to equation (3) with $\lambda \neq 0$ the initial condition

$$
p(t, 0)=f(t) \quad\left(t \in\left(0, t_{0}\right)\right)
$$

is added, to equation (3) with $\lambda \geq 0$ further the condition

$$
p(0, \tau)=\varphi(\tau) \quad(\tau \in(0, T))
$$




\section{Existence and uniqueness for equation (1)}

In equation (1) for $(t, \tau) \in R=\left(0, T_{0}\right) \times(0, T)$ we write the integral $I_{0}$ in the form

$$
I_{0}[p](t, \tau)=\int_{0}^{T_{0}-t} p(s, \cdot) * p(s+t, \cdot) d s
$$

where $*$ denotes convolution with respect to $\tau$. This integral is the quadratic form of the bilinear operator

$$
B\left[p_{1}, p_{2}\right](t, \tau) \equiv I_{0}\left[p_{1}, p_{2}\right](t, \tau)=\int_{0}^{T_{0}-t} p_{1}(s, \cdot) * p_{2}(s+t, \cdot) d s .
$$

For operator equations of the form $p+B[p, p]=h$ with bilinear operator $B$ there exist particular existence theorems in Banach spaces with a scale of norms which we apply in the form of $[2,3]$. We use the Banach spaces $L_{\alpha, \gamma}=L_{\alpha, \gamma}(R) \quad(1 \leq \alpha, \gamma \leq \infty)$ with mixed norms $\|\cdot\|_{\alpha, \gamma}$ and the scale of exponentially weighted norms

$$
\|p\|_{r} \equiv\|p\|_{\alpha, \gamma ; r}=\left\|e^{-r \tau} p\right\|_{\alpha, \gamma} \quad(r \geq 0)
$$

where, for $\alpha<\infty$,

$$
\begin{aligned}
& \|p\|_{\alpha, \gamma}=\left(\int_{0}^{T}\|p\|_{\alpha}^{\gamma}(\tau) d \tau\right)^{\frac{1}{\gamma}}(\gamma<\infty),\|p\|_{\alpha}(\tau)=\left(\int_{0}^{T_{0}}|p(t, \tau)|^{\alpha} d t\right)^{\frac{1}{\alpha}} \\
& \|p\|_{\alpha, \infty}=\sup \operatorname{ess}_{\tau}\|p\|_{\alpha}(\tau)
\end{aligned}
$$

and analogously, for $\alpha=\infty,\|p\|_{\infty, \gamma}(\gamma<\infty)$ and $\|p\|_{\infty, \infty}=\sup _{\operatorname{ess}_{\tau, t}}|p(t, \tau)|$. We notice that we always assume $T<\infty$ so that the weighted norms $\|\cdot\|_{\alpha, \gamma ; r}$ for any $r \geq 0$ are equivalent norms in $L_{\alpha, \gamma}$.

For operator (7) we have by Hölder's inequality the estimates

$$
\begin{aligned}
\left|B\left[p_{1}, p_{2}\right](t, \tau)\right| & \leq \int_{0}^{\tau} \int_{0}^{T_{0}-t} 1 \cdot\left|p_{1}(s, \sigma)\right|\left|p_{2}(s+t, \tau-\sigma)\right| d s d \sigma \\
& \leq\left(T T_{0}\right)^{\frac{1}{\beta}}\left(\int_{0}^{\tau} \int_{0}^{T_{0}-t}\left|p_{1}(s, \sigma)\right|^{\alpha}\left|p_{2}(s+t, \tau-\sigma)\right|^{\alpha} d s d \sigma\right)^{\frac{1}{\alpha}}
\end{aligned}
$$

where $\beta=\frac{\alpha}{\alpha-1}$ with $\alpha \geq 1$ and hence, for $\gamma \geq \alpha$,

$$
\begin{aligned}
\left\|B\left[p_{1}, p_{2}\right]\right\|_{\alpha, \gamma ; r}^{\gamma} & \left(T T_{0}\right)^{\frac{\gamma}{\beta}} \int_{0}^{T} e^{-\gamma r \tau}\left[\int _ { 0 } ^ { \tau } \left\{\int_{0}^{T_{0}}\left|p_{1}(s, \sigma)\right|^{\alpha} d s\right.\right. \\
\leq & \left.\left.\int_{0}^{T_{0}}\left|p_{2}\left(s^{\prime}, \tau-\sigma\right)\right|^{\alpha} d s^{\prime}\right\} d \sigma\right]^{\frac{\gamma}{\alpha}} d \tau \\
\leq & \left(T T_{0}\right)^{\frac{\gamma}{\beta}}\left\|p_{1}\right\|_{\alpha, \gamma ; r}^{\gamma}\left\|p_{2}\right\|_{\alpha, 1 ; r}^{\gamma}
\end{aligned}
$$


by Young's inequality with respect to $\tau$. In view of the inequalities

$$
\begin{aligned}
\|p\|_{\alpha, 1 ; r} & \leq T^{\frac{1}{\delta}}\|p\|_{\alpha, \gamma ; r} \\
\|p\|_{\alpha, 1 ; r} & \leq\left(\frac{1}{\delta r}\right)^{\frac{1}{\delta}}\|p\|_{\alpha, \gamma}
\end{aligned}
$$

where $\delta=\frac{\gamma}{\gamma-1}$, applied for $p=p_{2}$, this yields the desired inequalities

$$
\begin{aligned}
\left\|B\left[p_{1}, p_{2}\right]\right\|_{\alpha, \gamma ; r} & \leq N_{1}\left\|p_{1}\right\|_{\alpha, \gamma ; r}\left\|p_{2}\right\|_{\alpha, \gamma ; r} \\
\left\|B\left[p_{1}, p_{2}\right]\right\|_{\alpha, \gamma ; r} & \leq N_{2}(r)\left\|p_{1}\right\|_{\alpha, \gamma ; r}\left\|p_{2}\right\|_{\alpha, \gamma}
\end{aligned}
$$

with $N_{1}=\left(T T_{0}\right)^{\frac{1}{\beta}} T^{\frac{1}{\delta}}$ and $N_{2}(r)=\left(T T_{0}\right)^{\frac{1}{\beta}}\left(\frac{1}{\delta r}\right)^{\frac{1}{\delta}}$, and analogously for $p_{1}$ and $p_{2}$ interchanged where $1 \leq \alpha \leq \gamma \leq \infty$, with $\frac{1}{\beta}=0$ for $\alpha=1$ and $\frac{1}{\delta}=0$ for $\gamma=1$.

If $\gamma>1$, then $1 \leq \delta<\infty$, so that $N_{2}(r) \rightarrow 0$ as $r \rightarrow \infty$. By the existence theorem in $[2,3]$ then a uniquely determined solution $p \in L_{\alpha, \gamma}$ to equation (1) exists for any $h \in L_{\alpha, \gamma}$. This solution can be calculated by iteration and it is stable with respect to perturbations of $h$ satisfying a local Lipschitz condition of the form

$$
\left\|p_{1}-p_{2}\right\|_{\alpha, \gamma} \leq \Lambda\left(\left\|h_{1}\right\|_{\alpha, \gamma},\left\|h_{2}\right\|_{\alpha, \gamma}\right)\left\|h_{1}-h_{2}\right\|_{\alpha, \gamma}
$$

with some continuous increasing function $\Lambda(\cdot, \cdot)[2]$ and

$$
\left\|p_{1}-p_{2}\right\|_{\alpha, \gamma ; r} \leq 2\left\|h_{1}-h_{2}\right\|_{\alpha, \gamma ; r}
$$

for sufficiently large $r$ and sufficiently small $\left\|h_{1}-h_{2}\right\|_{\alpha, \gamma ; r}[3]$, where $p_{j} \in L_{\alpha, \gamma}$ are the solutions to equation (1) for $h_{j} \in L_{\alpha, \gamma}(j=1,2)$.

For $\alpha=1$ the coefficients $N_{1}, N_{2}$ in (9) are independent of $T_{0}$ so that the existence theorem also holds for equation (1) with integral $I$ from (4) in the strip $S=(0, \infty) \times(0, T)$ in this case.

Theorem 1. Let $1 \leq \alpha \leq \gamma, \gamma>1$ in the case of $R=\left(0, T_{0}\right) \times(0, T)$ and $\alpha=1, \gamma>1$ in the case of $S=(0, \infty) \times(0, T)$. Then for any $h \in L_{\alpha, \gamma}$ equation (1) possesses in $R$ as well as in $S$ a uniquely determined solution $p \in L_{\alpha, \gamma}$. This solution can be calculated by iteration and it is stable with respect to a perturbation of data $h$ in $L_{\alpha, \gamma}$.

Corollary 1. If $\alpha \geq 2, \gamma \geq 1$, then by Hölder's and Young's inequalities we get inequalities (9) with $N_{1}=T_{0}^{\frac{1}{\beta}} T^{\frac{1}{\delta}}$ and $N_{2}(r)=T_{0}^{\frac{1}{\beta}}\left(\frac{1}{\delta r}\right)^{\frac{1}{\delta}}$ yielding the existence of a solution $p \in L_{\alpha, \gamma}$ to equation (1) in $R$ under these assumptions, too. 


\section{Existence and uniqueness for equation (2)}

Equation (2) with initial condition (5) is reduced to the equation

$$
p(t, \tau)+C_{\mu}[p](t, \tau)=g_{\mu}(t, \tau) \quad((t, \tau) \in R)
$$

where

$$
\begin{aligned}
C_{\mu}[p](t, \tau) & =e^{-\mu \tau} * I_{0}[p](t, \tau) \\
g_{\mu}(t, \tau) & =e^{-\mu \tau} f(t)+\int_{0}^{\tau} e^{-\mu(\tau-\sigma)} h(t, \sigma) d \sigma
\end{aligned}
$$

and $*$ denotes again convolution with respect to $\tau$. The bilinear operator corresponding to (10) has the form

$$
C_{\mu}\left[p_{1}, p_{2}\right](t, \tau)=e^{-\mu \tau} * B\left[p_{1}, p_{2}\right](t, \tau) .
$$

We again assume $1 \leq \alpha \leq \gamma$ and estimate

$$
\begin{aligned}
\left|C_{\mu}\left[p_{1}, p_{2}\right](t, \tau)\right| & \leq \int_{0}^{\tau} 1 \cdot e^{-\mu \sigma}\left|B\left[p_{1}, p_{2}\right](t, \tau-\sigma)\right| d \sigma \\
& \leq T^{\frac{1}{\beta}}\left(\int_{0}^{\tau} e^{-\alpha \mu \sigma}\left|B\left[p_{1}, p_{2}\right](t, \tau-\sigma)\right|^{\alpha} d \sigma\right)^{\frac{1}{\alpha}}
\end{aligned}
$$

implying

$$
\begin{aligned}
\left\|C_{\mu}\left[p_{1}, p_{2}\right]\right\|_{\alpha, \gamma ; r}^{\gamma} & \\
\leq & T^{\frac{\gamma}{\beta}} \int_{0}^{T}\left[\int _ { 0 } ^ { \tau } \left\{e^{-\alpha(r+\mu) \sigma} e^{-\alpha r(\tau-\sigma)}\right.\right. \\
& \left.\left.\times \int_{0}^{T_{0}}\left|B\left[p_{1}, p_{2}\right](t, \tau-\sigma)\right|^{\alpha} d t\right\} d \sigma\right]^{\frac{\gamma}{\alpha}} d \tau \\
\leq & T^{\frac{\gamma}{\beta}}\left\|e^{-\mu \tau}\right\|_{\alpha, r}^{\gamma}\left\|B\left[p_{1}, p_{2}\right]\right\|_{\alpha, \gamma ; r}^{\gamma} .
\end{aligned}
$$

This yields the estimation

$$
\left\|C_{\mu}\left[p_{1}, p_{2}\right]\right\|_{\alpha, \gamma ; r} \leq T^{\frac{1}{\beta}}\left(\frac{1}{\alpha(r+\mu)}\right)^{\frac{1}{\alpha}}\left\|B\left[p_{1}, p_{2}\right]\right\|_{\alpha, \gamma ; r} \leq T^{\frac{1}{\beta}}\left\|B\left[p_{1}, p_{2}\right]\right\|_{\alpha, \gamma ; r}
$$

for $r>\max (1-\mu, 0)$. Hence by $(9)$ we obtain analogous inequalities for $C_{\mu}$

with coefficients $\hat{N}_{1}=T_{0}^{\frac{1}{\beta}} T^{\frac{2}{\beta}+\frac{1}{\delta}}$ and $\hat{N}_{2}(r)=T_{0}^{\frac{1}{\beta}} T^{\frac{2}{\beta}}\left(\frac{1}{\alpha(r+\mu)}\right)^{\frac{1}{\alpha}}\left(\frac{1}{\delta r}\right)^{\frac{1}{\delta}}$ where $\hat{N}_{2}(r) \rightarrow 0$ as $r \rightarrow \infty$.

So we have existence of a unique (mild) solution $p \in L_{\alpha, \gamma} \quad(1 \leq \alpha \leq \gamma)$ to equation (2) with condition (5) for any $f, h$ with $g_{\mu} \in L_{\alpha, \gamma}$. The last relation holds if $f \in L_{\alpha}$ and $h \in L_{\alpha, 1}$. If also $h \in L_{\alpha, \gamma}$, by equation (2) and inequality (9) 1 the solution $p \in L_{\alpha, \gamma}$ has a derivative $\frac{\partial p}{\partial \tau} \in L_{\alpha, \gamma}$ (strong solution).

For $\alpha=1$ the coefficients $\hat{N}_{1}, \hat{N}_{2}$ are again independent of $T_{0}$ and the existence theorem for equation (2) with condition (5) holds also in the strip $S=(0, \infty) \times(0, T)$. 
Theorem 2. Let $1 \leq \alpha \leq \gamma$ in the case of $R=\left(0, T_{0}\right) \times(0, T)$ and $1=\alpha \leq \gamma$ in the case of $S=(0, \infty) \times(0, T)$. Then for any $h \in L_{\alpha, \gamma}$ and $f \in L_{\alpha}$ equation (2) with condition (5) possesses in $R$ as well as in $S$ a uniquely determined solution $p \in L_{\alpha, \gamma}$ with derivative $\frac{\partial p}{\partial \tau} \in L_{\alpha, \gamma}$.

Remark. In general, $\frac{\partial p}{\partial t}$ does not exist. But if, in addition, $f$ and $h$ have derivatives $f^{\prime} \in L_{\alpha}$ and $\frac{\partial h}{\partial t} \in L_{\alpha, \gamma}$, differentiating (10) with respect to $t$ yields a linear Volterra integral equation of the second kind for the derivative $\frac{\partial p}{\partial t}$, with summable kernel and right-hand side from $L_{\alpha, \gamma}$ for given $p \in L_{\alpha, \gamma}$. From this $\frac{\partial p}{\partial t} \in L_{\alpha, \gamma}$ follows.

\section{Equation (3) with $\lambda=0$}

Equation (3) for $\lambda=0$ with initial condition (6) reduces to the equation

$$
p(t, \tau)+J_{0}[p](t, \tau)=h_{0}(t, \tau) \quad((t, \tau) \in R)
$$

where

$$
\begin{aligned}
J_{0}[p](t, \tau) & =e^{-\mu t} \circledast I_{0}[p](t, \tau) \\
h_{0}(t, \tau) & =e^{-\mu t} \varphi(\tau)+\int_{0}^{t} e^{-\mu(t-s)} h(s, \tau) d \tau
\end{aligned}
$$

and $\circledast$ denotes convolution with respect to $t$. The bilinear operator corresponding to (11) is given by

$$
D_{\mu}\left[p_{1}, p_{2}\right](t, \tau)=e^{-\mu t} \circledast B\left[p_{1}, p_{2}\right](t, \tau) .
$$

Applying Young's inequality with respect to $t$ we obtain

$$
\begin{aligned}
\int_{0}^{T_{0}}\left|D_{\mu}\left[p_{1}, p_{2}\right](t, \tau)\right|^{\alpha} d t & =\int_{0}^{T_{0}}\left|\int_{0}^{t} e^{-\mu(t-s)} B\left[p_{1}, p_{2}\right](s, \tau) d s\right|^{\alpha} d t \\
& \leq\left\|e^{-\mu t}\right\|_{\alpha}^{\alpha}\left(\int_{0}^{T_{0}}\left|B\left[p_{1}, p_{2}\right](s, \tau)\right| d s\right)^{\alpha}
\end{aligned}
$$

which yields the estimate

$$
\left\|D_{\mu}\left[p_{1}, p_{2}\right]\right\|_{\alpha, \gamma ; r} \leq A_{\alpha}\left\|B\left[p_{1}, p_{2}\right]\right\|_{1, \gamma ; r}
$$

where

$$
A_{\alpha}= \begin{cases}T_{0}^{\frac{1}{\alpha}} & \text { for } \mu=0 \\ \left(\frac{1}{\mu \alpha}\right)^{\frac{1}{\alpha}} & \text { for } \mu>0 \\ \left(\frac{1}{|\mu| \alpha}\right)^{\frac{1}{\alpha}} e^{|\mu| T_{0}} & \text { for } \mu<0\end{cases}
$$


Observing (9), for $1 \leq \alpha \leq \gamma$ we further have

$$
\begin{aligned}
\left\|D_{\mu}\left[p_{1}, p_{2}\right]\right\|_{\alpha, \gamma ; r} \leq A_{\alpha} T^{\frac{1}{\delta}}\left\|p_{1}\right\|_{1, \gamma ; r}\left\|p_{2}\right\|_{1, \gamma ; r} \\
\left\|D_{\mu}\left[p_{1}, p_{2}\right]\right\|_{\alpha, \gamma ; r} \leq A_{\alpha}\left(\frac{1}{\delta r}\right)^{\frac{1}{\delta}}\left\|p_{1}\right\|_{1, \gamma ; r}\left\|p_{2}\right\|_{1, \gamma}
\end{aligned}
$$

and analogously with interchanged $p_{1}$ and $p_{2}$. Finally, by Hölder's inequality, $\|p\|_{1, \gamma ; r} \leq T_{0}^{\frac{1}{\beta}}\|p\|_{\alpha, \gamma ; r} \quad(r \geq 0)$. Therefore, inequalities (9) hold for $D_{\mu}$ with the coefficients

$$
\begin{aligned}
\tilde{N}_{1} & =A_{\alpha} T_{0}^{\frac{2}{\beta}} T^{\frac{1}{\delta}} \\
\tilde{N}_{2}(r) & =A_{\alpha} T_{0}^{\frac{2}{\beta}}\left(\frac{1}{\delta r}\right)^{\frac{1}{\delta}} \quad(r>0)
\end{aligned}
$$

where $\tilde{N}_{2}(r) \rightarrow 0$ as $r \rightarrow \infty$ if $\gamma>1$.

So we obtain the existence of a unique (mild) solution $p \in L_{\alpha, \gamma} \quad(1 \leq$ $\alpha \leq \gamma, \gamma>1$ ) to equation (3) for $\lambda=0$ with condition (6) for any $\varphi, h$ with

$h_{0} \in L_{\alpha, \gamma}$ in equation (11). This is fulfilled if $\varphi \in L_{\gamma}$ and $h \in L_{1, \gamma}$. If also $h \in L_{\alpha, \gamma}$, by equation (3) with $\lambda=0$ and inequality $(9)_{1}$ the solution $p \in L_{\alpha, \gamma}$ has a derivative $\frac{\partial p}{\partial t} \in L_{\alpha, \gamma}$ (strong solution).

In the particular case $\mu>0$, for $\alpha=1$ the coefficients $\tilde{N}_{1}$ and $\tilde{N}_{2}$ in (12) are independent of $T_{0}$ and the result is valid for equation (3) with $\lambda=0$ in the strip $S=(0, \infty) \times(0, T)$, too.

Theorem 3. Let $1 \leq \alpha \leq \gamma, \gamma>1$ in the case of $R=\left(0, T_{0}\right) \times(0, T)$ and $\alpha=1, \gamma>1$ in the case of $S=(0, \infty) \times(0, T)$. Then for any functions $h \in L_{\alpha, \gamma}$ and $\varphi \in L_{\gamma}$ equation (3) possesses, for $\lambda=0$ with condition (6) in $R$ and for $\lambda=0, \mu>0$ in $S$, a unique solution $p \in L_{\alpha, \gamma}$ with derivative $\frac{\partial p}{\partial t} \in L_{\alpha, \gamma}$.

Remark. If, in addition, the functions $\varphi$ and $h$ have derivatives $\dot{\varphi} \in L_{\gamma}$ and $\frac{\partial h}{\partial \tau} \in L_{\alpha, \gamma}$, then also $\frac{\partial p}{\partial \tau} \in L_{\alpha, \gamma}$.

\section{Equation (3) with $\lambda \neq 0$}

Equation (3) for $\lambda>0$ in $R=\left(0, T_{0}\right) \times(0, T)$ with initial conditions (5) and (6) is equivalent to the equations

$$
\begin{array}{ll}
p(t, \tau)+J_{1}[p](t, \tau)=h_{1}(t, \tau) & \left((t, \tau) \in R \cap\{(t, \tau): \tau>\lambda t\}=R_{1}\right) \\
p(t, \tau)+J_{2}[p](t, \tau)=h_{2}(t, \tau) & \left((t, \tau) \in R \cap\{(t, \tau): \tau<\lambda t\}=R_{2}\right)
\end{array}
$$


where

$$
\begin{aligned}
& J_{1}[p](t, \tau)=\int_{0}^{t} e^{-\mu(t-s)} I_{0}[p](s, \tau-\lambda[t-s]) d s \\
& J_{2}[p](t, \tau)=\frac{1}{\lambda} \int_{0}^{\tau} e^{-\frac{\mu}{\lambda}(\tau-\sigma)} I_{0}[p]\left(t-\frac{1}{\lambda}[\tau-\sigma], \sigma\right) d \sigma
\end{aligned}
$$

and

$$
\begin{aligned}
& h_{1}(t, \tau)=e^{-\mu t} \varphi(\tau-\lambda t)+\int_{0}^{t} e^{-\mu(t-s)} h(s, \tau-\lambda[t-s]) d s \\
& h_{2}(t, \tau)=e^{-\frac{\mu}{\lambda} \tau} f\left(t-\frac{\tau}{\lambda}\right)+\frac{1}{\lambda} \int_{0}^{\tau} e^{-\frac{\mu}{\lambda}(\tau-\sigma)} h\left(t-\frac{1}{\lambda}[\tau-\sigma], \sigma\right) d \sigma .
\end{aligned}
$$

We consider equations (13) in the spaces $L^{1}=L_{1,1}$ and $L^{\infty}=L_{\infty, \infty}$. For the corresponding bilinear operator $D_{\lambda, \mu}$ we have

$$
\left\|D_{\lambda, \mu}\left[p_{1}, p_{2}\right]\right\|_{1,1 ; r}=W_{1}+W_{2}
$$

with

$$
W_{k}=\iint_{R_{k}} e^{-r \tau}\left|J_{k}\left[p_{1}, p_{2}\right](t, \tau)\right| d t d \tau \quad(k=1,2)
$$

where $J_{k}\left[p_{1}, p_{2}\right]$ are defined by $(14)$ with $B\left[p_{1}, p_{2}\right]=I_{0}\left[p_{1}, p_{2}\right]$ instead of $I_{0}[p]$. We estimate

$$
\begin{aligned}
W_{1} & \leq \int_{0}^{\min \left(T_{0}, \frac{T}{\lambda}\right)} \int_{\lambda t}^{T} e^{-r \tau} \int_{0}^{t} e^{-\mu(t-s)}\left|B\left[p_{1}, p_{2}\right](s, \tau-\lambda(t-s))\right| d s d \tau d t \\
& =\int_{0}^{\min \left(T_{0}, \frac{T}{\lambda}\right)} \int_{0}^{T-\lambda t} e^{-r\left[\tau^{\prime}+\lambda t\right]} \int_{0}^{t} e^{-\mu(t-s)}\left|B\left[p_{1}, p_{2}\right]\left(s, \tau^{\prime}+\lambda s\right)\right| d s d \tau d t .
\end{aligned}
$$

Changing the order of integration with respect to $\tau^{\prime}$ and $t$ and using Young's inequality with respect to $t$, for $T_{0} \geq \frac{T}{\lambda}$ we obtain

$$
\begin{aligned}
W_{1} & \leq \int_{0}^{T} e^{-r \tau^{\prime}}\left[\int_{0}^{\frac{T-\tau^{\prime}}{\lambda}} e^{-(\lambda r+\mu) t} d t \int_{0}^{\frac{T-\tau^{\prime}}{\lambda}}\left|B\left[p_{1}, p_{2}\right]\left(t, \tau^{\prime}+\lambda t\right)\right| e^{-\lambda r t} d t\right] d \tau^{\prime} \\
& \leq \frac{1}{\lambda r+\mu} \int_{0}^{T} e^{-r \tau^{\prime}} \int_{0}^{\frac{T-\tau^{\prime}}{\lambda}}\left|B\left[p_{1}, p_{2}\right]\left(t, \tau^{\prime}+\lambda t\right)\right| e^{-\lambda r t} d t d \tau^{\prime} \\
& =\frac{1}{\lambda r+\mu} \int_{0}^{T} e^{-r \tau} \int_{0}^{\frac{\tau}{\lambda}}\left|B\left[p_{1}, p_{2}\right](t, \tau)\right| d t d \tau
\end{aligned}
$$

for $r>\max \left(0,-\frac{\mu}{\lambda}\right)$. Analogously, for $T_{0}<\frac{T}{\lambda}$ we have

$$
W_{1} \leq \frac{1}{\lambda r+\mu} \int_{0}^{T_{0}} \int_{\lambda t}^{T} e^{-r \tau}\left|B\left[p_{1}, p_{2}\right](t, \tau)\right| d \tau d t
$$


for $r>\max \left(0,-\frac{\mu}{\lambda}\right)$. Further, using now Young's inequality with respect to $\tau$ we get

$$
W_{2} \leq \frac{1}{\lambda r+\mu} \times \begin{cases}\int_{0}^{T} e^{-r \tau} \int_{\tau / \lambda}^{T_{0}}\left|B\left[p_{1}, p_{2}\right](t, \tau)\right| d t d \tau & \text { for } T_{0} \geq \frac{T}{\lambda} \\ \int_{0}^{T_{0}} \int_{0}^{\lambda t} e^{-r \tau} \mid B\left[p_{1}, p_{2}\right](t, \tau \mid d \tau d t & \text { for } T_{0}<\frac{T}{\lambda}\end{cases}
$$

Therefore, in both cases the estimation

$$
\left\|D_{\lambda, \mu}\left[p_{1}, p_{2}\right]\right\|_{1,1 ; r} \leq \frac{1}{\lambda r+\mu}\left\|B\left[p_{1}, p_{2}\right]\right\|_{1,1 ; r}
$$

for $r>\max \left(0,-\frac{\mu}{\lambda}\right)$ holds. Observing inequalities (9), the desired inequalities

$$
\begin{aligned}
\left\|D_{\lambda, \mu}\left[p_{1}, p_{2}\right]\right\|_{1,1 ; r} & \leq \bar{N}_{1}\left\|p_{1}\right\|_{1,1 ; r}\left\|p_{2}\right\|_{1,1 ; r} \\
\left\|D_{\lambda, \mu}\left[p_{1}, p_{2}\right]\right\|_{1,1 ; r} & \leq \bar{N}_{2}(r)\left\|p_{1}\right\|_{1,1 ; r}\left\|p_{2}\right\|_{1,1}
\end{aligned}
$$

follow where $\bar{N}_{1}=1$ and $\bar{N}_{2}(r)=\frac{1}{\lambda r+\mu} \quad\left(r>\max \left(0, \frac{1}{\lambda}-\frac{\mu}{\lambda}\right)\right)$.

So, for any functions $h, f, \varphi$ with $\left\{h_{1}, h_{2}\right\} \in L^{1}(R)$ equation (3) for $\lambda>0$ with conditions (5) and (6) has a uniquely determined solution $p \in L^{1}(R)$. The relation $\left\{h_{1}, h_{2}\right\} \in L^{1}(R)$ is fulfilled if $f \in L^{1}\left(0, T_{0}\right), \varphi \in L^{1}(0, T)$ and $h \in L^{1}(R)$. For $h \in L^{1}(R)$, by equation (3) and inequality (9) $)_{1}$ the solution $p$ possesses the directional derivative $\frac{\partial p}{\partial t}+\lambda \frac{\partial p}{\partial \tau} \in L^{1}(R)$. Since $\bar{N}_{1}$ and $\bar{N}_{2}$ are independent of $T_{0}$, this holds also in the strip $S=(0, \infty) \times(0, T)$.

In the space $L^{\infty}(R)$ we simpler have

$$
e^{-r \tau}\left|J_{k}\left[p_{1}, p_{2}\right](t, \tau)\right| \leq \frac{1}{\lambda r+\mu} \sup \underset{t, \tau}{\operatorname{ess}}\left[e^{-r \tau}\left|B\left[p_{1}, p_{2}\right](t, \tau)\right|\right]
$$

for $r>\max \left(0,-\frac{\mu}{\lambda}\right)$ and $k=1,2$ so that also

$$
\left\|D_{\lambda, \mu}\left[p_{1}, p_{2}\right]\right\|_{\infty, \infty ; r} \leq \frac{1}{\lambda r+\mu}\left\|B\left[p_{1}, p_{2}\right]\right\|_{\infty, \infty ; r}
$$

for $r>\max \left(0,-\frac{\mu}{\lambda}\right)$. Again by (9), in $L^{\infty}$ from (18) we obtain inequalities analogous to (17) with coefficients $\bar{N}_{1, \infty}=T_{0} T^{2}$ and $\bar{N}_{2, \infty}(r)=\frac{T_{0} T}{(\lambda r+\mu) r}$, $r>\max \left(0, \frac{1}{\lambda}-\frac{\mu}{\lambda}\right)$. This implies existence and uniqueness of the solution $p \in$ $L^{\infty}(R)$ to equation (3) for $\lambda>0$ with conditions (5) and (6) if $f \in L^{\infty}\left(0, T_{0}\right)$, $\varphi \in L^{\infty}(0, T)$ and $h \in L^{\infty}(R)$ ensuring $\left\{h_{1}, h_{2}\right\} \in L^{\infty}(R)$. In view of $h \in$ $L^{\infty}(R)$ the solution $p$ possesses the directional derivative $\frac{\partial p}{\partial t}+\lambda \frac{\partial p}{\partial \tau} \in L^{\infty}(R)$. 
Theorem 4. For any functions $f \in L^{1}, \varphi \in L^{1}$ and $h \in L^{1}$ equation (3) for $\lambda>0$ with conditions (5) and (6) possesses in $R=\left(0, T_{0}\right) \times(0, T)$ and $S=(0, \infty) \times(0, T)$ a unique solution $p \in L^{1}$. For this solution the directional derivative $\frac{\partial p}{\partial t}+\lambda \frac{\partial p}{\partial \tau} \in L^{1}$ exists.

Further, for any functions $f \in L^{\infty}\left(0, T_{0}\right), \varphi \in L^{\infty}(0, T)$ and $h \in L^{\infty}(R)$ equation (3) for $\lambda>0$ with conditions (5) and (6) possesses a unique solution $p \in L^{\infty}(R)$ satisfying $\frac{\partial p}{\partial t}+\lambda \frac{\partial p}{\partial \tau} \in L^{\infty}(R)$.

Corollary 2. If $f \in C\left[0, T_{0}\right]$ and $\varphi \in C[0, T]$ with $f(0)=\varphi(0)$ and if $h \in C(\bar{R})$, then $p \in C(\bar{R})$ with $\frac{\partial p}{\partial t}+\lambda \frac{\partial p}{\partial \tau} \in C(\bar{R})$ for the solution $p$ of equation (3) for $\lambda>0$ with conditions (5) and (6).

Remark. If the functions $f, \varphi, h$ have derivatives $f^{\prime}, \dot{\varphi}, \frac{\partial h}{\partial t}, \frac{\partial h}{\partial \tau}$ in $L^{1}$ or $L^{\infty}$, then the solutions $p$ have derivatives $\frac{\partial p}{\partial t}$ and $\frac{\partial p}{\partial \tau}$.

Finally, we deal with equation (3) for $\lambda<0$ with initial condition (5) in $S=(0, \infty) \times(0, T)$. The problem is reduced to the equation

$$
p(t, \tau)+J_{2}[p](t, \tau)=h_{2}(t, \tau) \quad((t, \tau) \in S)
$$

where $J_{2}[p]$ and $h_{2}$ are given by formulas (14) - (15). We consider equation (19) in the space $L^{1}(S)$. As above we estimate

$$
\left\|D_{\lambda, \mu}\left[p_{1}, p_{2}\right]\right\|_{1,1 ; r}=\int_{0}^{T} \int_{0}^{\infty} e^{-r \tau}\left|J_{2}\left[p_{1}, p_{2}\right](t, \tau)\right| d t d \tau
$$

by

$$
\left\|D_{\lambda, \mu}\left[p_{1}, p_{2}\right]\right\|_{1,1 ; r} \leq \frac{1}{|\lambda| r-\mu}\left\|B\left[p_{1}, p_{2}\right]\right\|_{1,1 ; r}
$$

for $r>\max \left(0, \frac{\mu}{|\lambda|}\right)$ and obtain existence and uniqueness of the solution $p \in$ $L^{1}(S)$ for any $f \in L^{1}\left(\mathbb{R}_{+}\right)$and $h \in L^{1}(S)$. This solution possesses the directional derivative $\frac{\partial p}{\partial t}+\lambda \frac{\partial p}{\partial \tau} \in L^{1}(S)$.

Theorem 5. For any functions $f \in L^{1}\left(\mathbb{R}_{+}\right)$and $h \in L^{1}(S)$ equation (3) for $\lambda<0$ with initial condition (5) possesses a unique solution $p \in L^{1}(S)$ satisfying $\frac{\partial p}{\partial t}+\lambda \frac{\partial p}{\partial \tau} \in L^{1}(S)$.

Remark. If the functions $f$ and $h$ have derivatives $f^{\prime} \in L^{1}\left(\mathbb{R}_{+}\right)$and $\frac{\partial h}{\partial t}, \frac{\partial h}{\partial \tau} \in L^{1}(S)$, then the solutions $p$ have derivatives $\frac{\partial p}{\partial t}, \frac{\partial p}{\partial \tau} \in L^{1}(S)$. 


\section{Generalizations}

The above existence and uniqueness results also hold for equations (1) - (3) with the generalized correlation-convolution integral

$$
I_{1}[p](t, \tau)=\int_{0}^{\tau} \int_{0}^{T_{0}-t} G_{1}[p](s, \sigma) G_{2}[p](s+t, \tau-\sigma) d s d \sigma
$$

instead of $I_{0}[p]$, where the operators $G_{k}: L_{\alpha, \gamma} \rightarrow L_{\alpha, \gamma}$ satisfy Lipschitz conditions of the form

$$
\left\|G_{k}\left[p_{1}\right]-G_{k}\left[p_{2}\right]\right\|_{\alpha, \gamma ; r} \leq M_{k}\left(\left\|p_{1}\right\|_{\alpha, \gamma ; r},\left\|p_{2}\right\|_{\alpha, \gamma ; r}\right)\left\|p_{1}-p_{2}\right\|_{\alpha, \gamma ; r}
$$

with continuous increasing functions $M_{k}(\cdot, \cdot) \quad(k=1,2)$ (cf. [2]). Also, obviously, in the correlation-convolution integral an additional bounded measurable kernel $K=K(s, t ; \sigma, \tau)$ as a factor can be present.

Further, the method of weighted norms can be used to a direct investigation of the uniqueness of the solutions to equations (1) - (3). For instance, if $p_{j}(j=1,2)$ are solutions of equation $(1)$ in $L^{1}(S)$ for a fixed right-hand side $h \in L^{1}(S)$, their difference $p=p_{1}-p_{2} \in L^{1}(S)$ obeys the equation

$$
p(t, \tau)+\int_{0}^{\tau} \int_{0}^{\infty} k(s, t ; \tau-\sigma) p(s, \sigma) d s d \sigma=0 \quad(t>0)
$$

with the kernel

$$
k(s, t ; \sigma)= \begin{cases}p_{1}(s+t, \sigma)+p_{2}(s-t, \sigma) & \text { for } s>t \\ p_{1}(s+t, \sigma) & \text { for } s<t\end{cases}
$$

and the function $p_{r}(t, \tau)=e^{-r \tau} p(t, \tau) \quad(r \geq 0)$ obeys an equation analogous to $(20)$ with the kernel $k_{r}(s, t ; \sigma)=e^{-r \sigma} k(s, t ; \sigma)$. Hence we have uniqueness of the solution to equation $(1)$ in $L^{1}(S)$ if

$$
\sup _{s} \int_{0}^{\infty} \int_{0}^{T} e^{-r \tau}|k(s, t ; \tau)| d \tau d t<1
$$

for sufficiently large $r>0$, which is fulfilled if

$$
\sup _{s} \int_{0}^{T} \frac{1}{\rho^{\gamma}(\tau)}\left(\int_{0}^{\infty}|k(s, t ; \tau)| d t\right)^{\gamma} d \tau<\infty
$$

with a non-negative function $\rho \in L^{\delta}(0, T)$ with $\delta=\frac{\gamma}{\gamma-1}$ for $\gamma>1$, and with a function $\rho \in C_{0}[0, T]$, i.e. $\rho \in C[0, T]$ satisfying $\rho(0)=0$, for $\gamma=1$. 
In view of $(21)$,

$$
\int_{0}^{\infty}|k(s, t ; \tau)| d t \leq \int_{0}^{\infty}\left|p_{1}(t, \tau)\right| d t+\int_{0}^{\infty}\left|p_{2}(t, \tau)\right| d t
$$

and condition (22) is fulfilled if $p_{j} \in L^{1}(S) \quad(j=1,2)$ satisfy the inequality

$$
\int_{0}^{T} \frac{1}{\rho^{\gamma}(\tau)}\left(\int_{0}^{\infty}|p(t, \tau)| d t\right)^{\gamma} d \tau<\infty
$$

for $1 \leq \gamma<\infty$, with $\rho \in L^{\delta}(0, T)$ for $1<\gamma<\infty$ or $\rho \in C_{0}[0, T]$ for $\gamma=1$, or if

$$
\sup _{\tau}\left[\frac{1}{\rho(\tau)} \int_{0}^{\infty}|p(t, \tau)| d t\right]<\infty
$$

with $\rho \in L^{1}(0, T)$ for $\gamma=\infty$. The uniqueness criteria (23) and (24) are some generalizations to the uniqueness assertion in Theorem 1.

In analogous manner, for equation (3) with $\lambda=0$ and condition (6) we obtain uniqueness of the solution $p \in L^{1}(S)$ if

$$
\int_{0}^{T} \frac{1}{\rho^{\gamma}(\tau)}\left(\int_{0}^{\tau} e^{\kappa t}|p(t, \tau)| d t\right)^{\gamma} d \tau<\infty \quad(\gamma \geq 1)
$$

or

$$
\sup _{\tau}\left[\frac{1}{\rho(\tau)} \int_{0}^{\infty} e^{\kappa t}|p(t, \tau)| d t\right]<\infty
$$

with a function $\rho$ as in (23), (24) and the parameter $\kappa=0$ for $\mu>0$ and $\kappa>|\mu|$ for $\mu \geq 0$. This generalizes the uniqueness assertion in Theorem 3 .

\section{References}

[1] Gladwell, G. M. L.: Inverse Problems in Scattering. Dordrecht: Kluwer Acad. Publ. 1993.

[2] Janno, J.: Nonlinear equations with operators satisfying Lipschitz conditions in scales. Z. Anal. Anw. 18 (1999), $287-295$.

[3] Janno, J. and L. von Wolfersdorf: Identification of weakly singular memory kernels in heat conduction. Z. Angew. Math. Mech. (ZAMM) 77 (1997), 243 $-257$.

[4] Nussbaum, R. D.: A quadratic integral equation. Annali di Pisa 7 (1980), 375 -480 .

[5] Nussbaum, R. D.: A quadratic integral equation II. Indiana Univ. Math. J. 30 (1981), $871-906$. 
[6] Pimbley, G. Jr.: Positive solutions of a quadratic integral equation. Arch. Rat. Mech. Anal. 24 (1967), $107-127$.

[7] Ramalho, R.: Existence and uniqueness theorems for a nonlinear integral equation. Math. Ann. 221 (1976), $35-44$.

[8] von Wolfersdorf, L.: Einige Klassen quadratischer Integralgleichungen. Sitzber. Sächs. Akad. Wiss. Leipzig, Math.-naturwiss. Klasse 128 (2000), Heft 2.

[9] Wertheim, M. S.: Analytic solutions of the Percus-Yevick equation. J. Math. Phys. 5 (1964), $643-651$.

Received 16.04.2003 